\title{
A method for the establishment of a cell line with stable expression of the GFP-LC3 reporter protein
}

\author{
DONG HU, JING WU* ${ }^{*}$ LIFA XU, RONGBO ZHANG ${ }^{*}$ and LIPING CHEN \\ Department of Medical Immunology, Medical School, Anhui University of Science and Technology; \\ Institute of Infection and Immunology, Anhui University of Science and Technology, \\ Huainan, Anhui 232001, P.R. China
}

Received March 3, 2012; Accepted July 2, 2012

DOI: $10.3892 / \mathrm{mmr} .2012 .988$

\begin{abstract}
As the function of autophagy becomes evident in a number of diseases, including cancer and infection, it is crucial to construct macrophage cell lines with stable expression of the microtubule-associated protein light chain 3 (GFP-LC3). In this study, a mouse LC3 open-reading frame was amplified by RT-PCR, and cloned into the pEGFP-C1 plasmid for expression of the GFP-LC3 fusion protein. The recombinant plasmid was transfected into RAW264.7 cells using Lipofectamine 2000 reagent and stably transfected clones were selected by G418 screening. Autophagic puncta formation was observed by fluorescense microscopy. Additionally, we found that starvation treatment induced a significant increase in the number of autophagosomes, while wortmannin treatment significantly repressed the formation of autophagosomes. This study indicated that the RAW264.7 cell line stably expressing GFP-LC3 is available for use in a GFP-LC3 puncta formation assay, and may contribute to basic investigations of autophagic function or drug screening targeted at autophagy.
\end{abstract}

\section{Introduction}

Recent studies have identified autophagy, originally observed in yeast, as a highly evolutionarily conserved mechanism universally present in all eukaryotic cells (1). Certain overaggregations of intracellular proteins, organelles and pathogens are too large to be effectively degraded by the proteasome. At present, autophagy is the only known cell biological pathway able to eliminate these aggregates in order to maintain normal cell function. Functional deficit of autophagy contributes to a variety of diseases, including cancer, neurodegeneration and

Correspondence to: Professor Rongbo Zhang and Dr Jing Wu, Department of Medical Immunology, Medical School, Anhui University of Science and Technology, No. 25 Middle Dongshan Road, Huainan, Anhui 232001, P.R. China

E-mail: lory456@126.com; wujing8008@126.com

*Contributed equally

Key words: macrophage, autophagy, stable cell line, light chain 3 infectious diseases (2). The LC3 protein is an autophagosomal membrane marker that is usually dispersed throughout the cytoplasm. The cytoplasmic form of LC3 (LC3-I) is conjugated to phosphatidylethanolamine to produce the LC3-phosphatidylethanolamine conjugate (LC3-II), which is recruited to autophagosomal membranes under stimulation of starvation, lack of growth factors or immune factors, and certain intracellular pathogens, and is involved in the formation of autophagosomes.

Currently, as LC3-II is involved in the entire formation process of autophagosomal membranes, aggregation of GFP-LC3 fusion proteins in the autophagosome observed under fluorescence microscopy has become the most widely used method to evaluate the occurrence of autophagy (3). One of the effective mechanisms that macrophages, important immune cells, use to kill intracellular pathogens, including Mycobacterium tuberculosis, depends on the autophagy pathway, which performs an immune defense role (4). Owing to the insufficient transfection rate of liposome-transfected cells, in this study, we screened macrophage cell lines with stable expression of the GFP-LC3 protein, which may aid in basic investigations examining the autophagic function of macrophages or act as the cellular platform for drug screening.

\section{Materials and methods}

Plasmids. The pEGFP-C1 plasmids and DH5 $\alpha$ bacterial strains were gifts from Professor Xinbing Yu (Zhongshan School of Medicine, Sun Yat-Sen University, China). As in previous studies (5-7), cDNA encoding the LC3 protein was obtained by RT-PCR amplification using the specific primers (sense, ttactcgagatatgccetccgaccggectttc and anti-sense, accg gatcctcagaagccgaaggtttcttg). The PCR products encoding the LC3 protein were digested using XhoI and BamHI restriction endonucleases (Fermentas, Canada). The products were then inserted into the corresponding sites of the pEGFP-C1 plasmid digested with the same restriction enzymes in order to construct the pEGFP-LC3 plasmid. The plasmids were then transformed into DH5 $\alpha$ bacterial strains to screen for the recombinant plasmid. These recombinants were identified by DNA sequencing. Plasmid DNA was prepared and purified using a plasmid maxi kit (Tiangen Biochemical Technology Ltd., Co., Beijing, China), according to the manufacturer's 
instructions, suspended in endotoxin-free physiologic saline, and stored at $-20^{\circ} \mathrm{C}$ until use.

Cell transfection and stable cell line screening. Transient transfections were performed using Lipofectamine 2000 reagent (Invitrogen, Carlsbad, CA, USA). RAW264.7 cells were cultured in DMEM medium (Gibco) containing 10\% fetal bovine serum (FBS) and antibiotics in $5 \% \mathrm{CO}_{2}$ at $37^{\circ} \mathrm{C}$. The growth rate of RAW264.7 cells was then observed under an inverted microscope. When the confluence reached $85 \%$, the cells were plated in 6-well plates and supplemented with $2 \mu \mathrm{g}$ plasmid per well [plasmid $(\mu \mathrm{g})$ : Lipofectamine $2000(\mu \mathrm{l})$ $=1: 3]$. Twenty-four hours after transfection, cells were digested with $0.25 \%$ trypsin and the cultures were transferred to the plates for further culture with DMEM medium containing $600 \mathrm{mg} / \mathrm{l} \mathrm{G} 418$ and 10\% FBS for 10 days. When the amount of resistant cell clones was observed, they were digested with $0.25 \%$ trypsin and then transferred to a new culture flask using an aseptic pipette for further culture. Subsequently, the pEGFP-LC3 recombinant plasmids or pEGFP empty plasmids and pEGFP-LC3 recombinant bacterium liquid PCR products were cut using specific enzymes. The fragments were then subjected to agarose gel electrophoresis.

Autophagy induction and suppression. When stable cell growth was observed, DMEM medium was blotted and cells were washed three times with PBS at $37^{\circ} \mathrm{C}$. The cells were then incubated in Earle's balanced salts solution (starve group) for $1 \mathrm{~h}$ at $37^{\circ} \mathrm{C}$, or were simultaneously added to wortmannin at a final concentration of $50 \mathrm{nM}$ (starve+wort group). After $1 \mathrm{~h}$, at least 200 GFP-positive cells were selected for observation under an inverted fluorescence microscope (x400) in order to count the number of GFP-LC3 punctas. Under fluorescence microscopy, several bright green fluorescent punctas were observed in the cells. One puncta was regarded as equal to one autophagosome. The results are presented as the average number of punctas per cell.

Statistical analysis. Data were presented as the mean \pm SD. ANOVA was used to compare the means of more than two samples. $\mathrm{P}<0.05$ was considered statistically significant.

\section{Results}

Successful construction of pEGFP-LC3 vector. The open reading frame of the cDNA coding for the mouse LC3 protein was $366 \mathrm{bp}$. The 366-bp cDNA products were obtained by RT-PCR amplification using mouse cDNA from transcriptase as templates with specific primers, and then the recombinant plasmid was constructed. The 366-bp product was also amplified by PCR using positive bacterium liquid as a template. To determine whether the pEGFP-LC3 vectors were constructed successfully, the recombinant plasimds were cut into fragments by restriction enzymes, and agarose gel electrophoresis was performed. We identified bands of the target gene at $366 \mathrm{bp}$ (Fig. 1). Additionally, the sequencing results revealed that the recombinant gene sequences were completely matched. sion of GFP-LC3. After the RAW264.7 cell lines screened

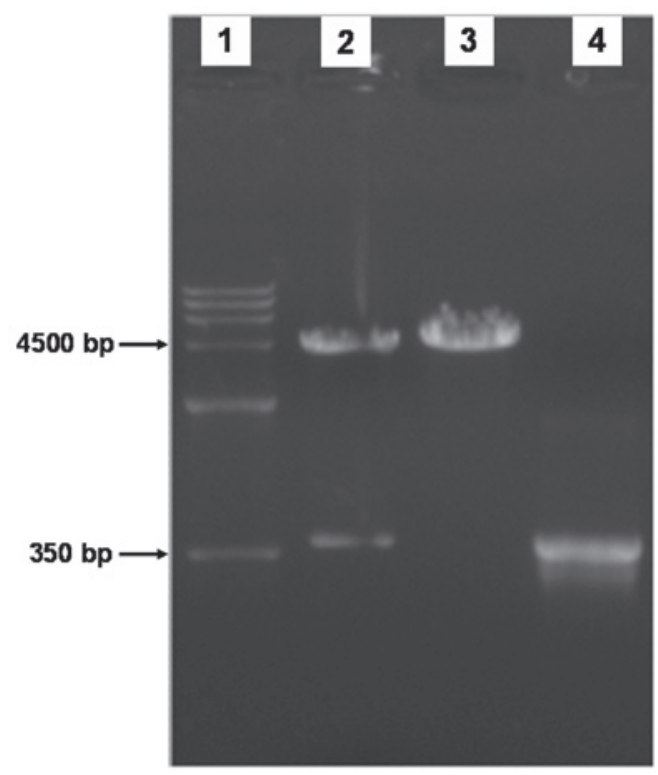

Figure 1. The pEGFP-LC3 recombinant and pEGFP empty plasmids were cut using specific enzymes. The fragments of pEGFP-LC3 recombinant plasmids (lane 2) or pEGFP empty plasmids (lane 3), DNA markers (lane 1) and pEGFP-LC3 recombinant bacterium liquid PCR (lane 4) were subjected to agarose gel electrophoresis. In comparison with the DNA markers (lane 1), the 366-bp band was observed in lanes 2 and 4, but not in lane 3 . LC3, light chain 3 .

by G418 were amplified and cultured, we found that the cell clones were able to stably express the GFP-LC3 fusion protein. Upon stimulation of starvation or lack of growth factor, the LC3 originally distributed in the cytoplasm was conjugated to the lipid phosphatidylethanolamine, forming LC3-II (the lipidated form). LC3-II was then recruited to the outer and inner membranes at each stage of autophagosome formation, and punctate aggregation occurred. In this study, we observed GFP-LC3 punctate structures in stably transfected cell lines by fluorescence microscopy. GFP-LC3 punctas of the majority of cells were homogeneously distributed in the cytoplasm in complete medium supplemented with FBS. Autophagic punctas were significantly increased in Earle's balanced salts solution, suggesting that treatment with starvation is able to effectively induce the formation of autophagosomes (Fig. 2). However, upon wortmannin treatment, the number of autophagosomes was evidently reduced, indicating that wortmannin is capable of suppressing autophagosome formation.

\section{Discussion}

In the recent years, the number of studies investigating autophagy in several areas, including cancer, immune factors, infection, inflammation and neurodegeneration, has been on the increase (2). Autophagy is a complex, dynamic process involved in the initation of autophagy, elongation and formation of the autophagosome, and autophagosome-lysosomal fusion $(8,9)$. Accurate detection of autophagy is important for the study of the biological functions of autophagy. Currently, although over 30 autophagy-related proteins (Atgs) have been identified, only LC3, the mammalian counterpart of yeast Atg8, is able to act as a marker of an autophagosome (3). LC3 

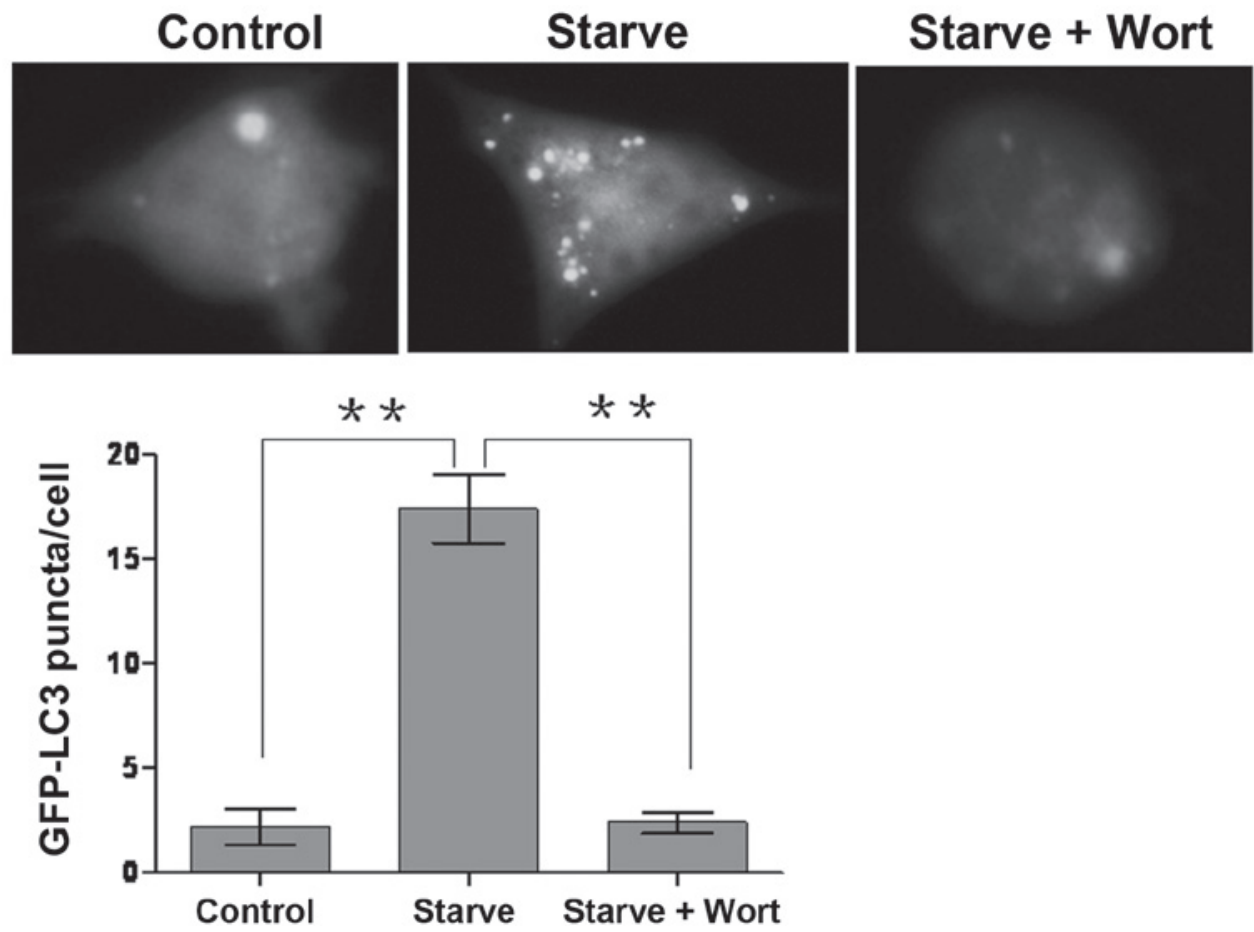

Figure 2. RAW264.7 cell line stable expression of GFP-LC3 is shown. RAW264.7 cells were transfected with pEGFP-LC3 plasmids. The RAW264.7 cells stably expressing GFP-LC3 (white) were incubated in complete media (control) and in Earle's balanced salts solution (starve) or simultaneously supplemented with wortmannin (starve+wort) for $1 \mathrm{~h}$. Upon starvation, the number of GFP-LC3 punctate structures was significantly increased compared to those incubated in complete media (control). However, the number of green fluorescent spots was evidently reduced in RAW264.7 cells incubated in Earle's balanced salts solution containing wortmannin (starve+wort). Quantitation shown represents the mean GFP-LC3 puncta per cell. Data are presented as the mean \pm SD $\left({ }^{* *} \mathrm{P}<0.05\right)$. Starve, starvation group; wort, wortmannin group; LC3, light chain 3.

occurs at all phases of autophagosome membrane formation, and therefore may be able to demonstrate the dynamic process of autophagosome formation.

LC3 undergoes two important changes in the process of autophagosome formation. The first is lipidation of the originally free LC3, which forms LC3-II bound to phosphatidylethanolamine $(\mathrm{PE})$. The other is the translocation of cytoplasmic LC3 distribution, which is localized in the autophagosome membrane. According to the characteristics of the conversion of LC3 to the lipidated LC3-II and the differences in mobility between LC3 and LC3-II in polyacrylamide gel electrophoresis (PAGE), we were able to detect the ratio of LC3/LC3-II by western blot analysis in order to indirectly reflect the formation of autophagosomes. When the formation of autophagosomes increased, the ratio of LC3/LC3-II was reduced; inversely, the ratio of LC3/LC-II increased $(3,10)$.

When the LC3 protein was combined with fluorescent tags, we were able to observe the formation of autophagosomes using autophagy puncta formation experiments based on the characteristics of LC3 attached to the autophagosome membrane. We then counted the number of fluorescent punctas per cell in the RAW264.7 cells and performed statistical quantitative analysis. In this study, LC3 proteins were combined with GFP tags. After the recombinant plasmids were successfully transfected into RAW264.7 cells, which had increased the expression of recombinant plasmids in 24-48 h, we counted the number of autophagosome punctas in the cells. However, cells instantaneously transfected with liposomes had two shortcomings; unstable efficiency of transfection leading to a reduction in cells effectively expressing GFP-LC3, and degradation of the recombinant plasmid resulting in a reduction in GFP-LC3 autophagy punctas. Therefore, we screened the RAW264.7 cell lines stably expressing GFP-LC3 using G418. The screened cell lines expressed the GFP-LC3 protein for an extended period of time. Following the starvation stimuli, the GFP-LC3 proteins, which originally homogeneously existed in the cytoplasm, aggregated to form bright autophagy puncta, suggesting that the cell line is useful in studies regarding the formation of autophagosomes.

Of note is that due to the dynamic process of autophagy, the number of autophagosomes at certain times should be the balanced result of autophagosome formation and conversion. Aggregation of the autophagosome is likely to reflect the induction of autophagy and the consequence of blockage of the downstream autophagy pathway. Therefore, it is insufficient to adequately represent the activity of autophagy using one or two methods (3). According to the complex characteristics of dynamic autophagy, it may be more accurate to evaluate autophagy function using autophagy flux; the increase in autophagy flux represents the enhancement of autophagy activity. Conversely, autophagy activity is reduced $(3,10,11)$. In conclusion, in this study, the stable cell lines provided a more reliable cell platform for detecting marked autophagy flux.

\section{Acknowledgements}

This study was supported by the Anhui Provincial Natural Science Foundation (No. 1208085QH162), AUST Grants (Dong $\mathrm{Hu}$ and Jing $\mathrm{Wu}$ ), the Colleges and Universities 
Education Grant of Anhui Province (no. 2008jp1042) and the National Natural Science Foundation Grants of China (no. 81041083 and no. 81172778).

\section{References}

1. Levine B, Mizushima N and Virgin HW: Autophagy in immunity and inflammation. Nature 469: 323-335, 2011.

2. He $\mathrm{C}$ and Klionsky DJ: Regulation mechanisms and signaling pathways of autophagy. Annu Rev Genet 43: 67-93, 2009.

3. Mizushima N, Yoshimori $\mathrm{T}$ and Levine B: Methods in mammalian autophagy research. Cell 140: 313-326, 2010.

4. Deretic V, Delgado M, Vergne I, Master S, De Haro S, Ponpuak M and Singh S: Autophagy in immunity against Mycobacterium tuberculosis: a model system to dissect immunological roles of autophagy. Curr Top Microbiol Immunol 335: 169-188, 2009.

5. Hu D, Wu J, Hu F, Yang Y, Liang C, Chen J, Wang L, Wang P, Wang $X, X u J, H u X$ and $Y u X$ : Stage and tissue specific differences in SjBMI1, a Polycomb protein in Schistosoma japonicum. Parasitol Res 106: 677-682, 2010.
6. Wu J, Hu D, Yang G, Zhou J, Yang C, Gao Y and Zhu Z: Down-regulation of BMI-1 cooperates with artemisinin on growth inhibition of nasopharyngeal carcinoma cells. J Cell Biochem 112: 1938-1948, 2011.

7. Hu D, Wu J, Tang X, Hu F, Yang Y, Du J, Ye S and Zhang R: Molecular cloning and tissue distribution of a Schistosoma japonicum gene encoding AMY-1. Mol Med Rep 4: 1267-1271, 2011.

8. Tang H, Da L, Mao Y, Li Y, Li D, Xu Z, Li F, Wang Y, Tiollais P, $\mathrm{Li}$ T and Zhao M: Hepatitis B virus $X$ protein sensitizes cells to starvation-induced autophagy via up-regulation of beclin 1 expression. Hepatology 49: 60-71, 2009.

9. Virgin HW and Levine B: Autophagy genes in immunity. Nat Immunol 10: 461-470, 2009.

10. Rubinsztein DC, Cuervo AM, Ravikumar B, Sarkar S, Korolchuk V, Kaushik S and Klionsky DJ: In search of an 'autophagomometer'. Autophagy 5: 585-589, 2009.

11. Ganley IG, Wong PM, Gammoh N and Jiang X: Distinct autophagosomal-lysosomal fusion mechanism revealed by thapsigargin-induced autophagy arrest. Mol Cell 42: 731-743, 2011. 